\title{
Skeletal Radiology: The Year in Review 2017
}

\author{
Daniel I. Rosenthal ${ }^{1} \cdot$ Mark J. Kransdorf $^{2} \cdot$ Thomas M. Link $^{3} \cdot$ Gunnar Astrom $^{4}$ \\ Published online: 22 December 2017 \\ (C) ISS 2017
}

In 2017, Skeletal Radiology published a total of 1678 pages, comprised of 97 scientific articles, 83 case reports, and 14 Test Yourself cases. There were also 21 review articles, 12 Browsers notes, five technical reports, and five letters to the editor. We also published meeting abstracts from the International Skeletal Society (ISS), and the affiliated Australasian Musculoskeletal Imaging Group (AMSIG) and Society of Skeletal Radiology (SSR). As we reflect upon this wealth of material, it is impossible to single out particular contributions based upon the quality or originality of the work. However, several papers stand out because they seem particularly timely or impactful. This is clearly a subjective matter, and your own choices may differ.

\section{Fascinating cases}

Skeletal Radiology has had a longstanding commitment to the publication of case reports. These often represent extremely exotic diagnoses, or unusual manifestations. Sometimes they are of exceptional interest. A report of a 66-year-old man with PPP syndrome (pancreatitis, panniculitis, and polyarthritis syndrome) by Kang et al. [1] is one such article. This

Daniel I. Rosenthal

dirosenthal@partners.org

Mark J. Kransdorf

kransdorf.mark@mayo.edu

Thomas M. Link

thomas.link@ucsf.edu

Gunnar Astrom

Gunnar.Astrom@radiol.uu.se

1 Massachusetts General Hospital, Boston, MA, USA

2 Mayo Clinic, Phoenix, AZ, USA

3 Department of Radiology and Biomedical Imaging, University of California, San Francisco, CA, USA

4 University of Uppsala, Uppsala, Sweden beautifully illustrated case report has already been cited twice in other publications. In other instances, a case report can be responsive to topics of interest more rapidly than a full scientific paper as in the report of successful cryoablation of a phosphaturic mesenchymal tumor causing osteomalacia [2].

\section{The practice of radiology}

Reflecting an international trend in medicine, the practice of Radiology has grown ever more complicated. Skeletal Radiology, like other radiology journals, has seen an increased level of interest in the way that radiology is practiced, and the effects that the changing health environment is having upon radiologists. We may not enjoy hearing about these issues, but they are undeniably important.

Pre-authorization is required by many US insurance companies to determine whether coverage will be provided for specific services. Pre-authorization is usually required for MRI. The usual justification for this practice (which is both annoying and costly) is that by going through the steps of preauthorization it will provide assurances that the examination is appropriate and necessary. Pierce et al. [3] reviewed 80 pediatric patients who were required to undergo pre-authorization prior to a knee MRI, and compared the findings on the examination to 44 patients who did not undergo pre-authorization. The authors found that there was no difference in the frequency with which surgically significant findings were present among the groups that had undergone preauthorization. The authors concluded that the pre-authorization process was a barrier to care that offered no medical benefit.

Another paper that deals with the current practice of radiology is "Prevalence of burnout among musculoskeletal radiologists" by Chew et al. [4]. It is unfortunately true that the extra-medical burdens (such as pre-authorizations) related to the practice of health care have been growing at a rapid rate. Unfortunately, this has led to emotional exhaustion, depersonalization, and perceptions of a lack of accomplishment, all symptoms of the syndrome of "burnout" according to Chew. 
These authors surveyed members of the Society of Skeletal Radiology and identified at least some feelings of professional "burn out" in over $80 \%$ of respondents. The percentages were higher in private practice than in academic settings, and were also higher than for other radiology specialists and other physicians. Published in April, this article has already been cited by other publications three times - a testament to the timeliness of its subject.

\section{Useful clinical insights}

Aneurysmal bone cyst has long been a management problem. The lesions can be large and may exhibit rapid growth. However, they are benign, and due to their hypocellular cystic nature, have quite a small amount of "tumor burden". Surgical management can be disabling and disfiguring and can be followed by recurrence. Therefore, the treatment sometimes seems worse than the disease. Because of this, a variety of non-surgical therapies have been used in an attempt to produce regression of the lesions, including several injectable agents. None have been completely satisfactory. In some instances, multiple injections are needed, and other lesions may fail to respond. Some of the most effective injectables are highly toxic to tissues, and produce marked inflammatory reactions and other complications. Chang et al. [5] report a series of eight patients with primary $\mathrm{ABC}$ who were treated with a combination of corticosteroid and calcitonin. Follow-up showed that half of the patients had substantial response, both clinically and on imaging studies, such that no further treatment was needed. Although this response rate is somewhat less than has been reported for more irritating substances (Ethibloc, doxycycline, alcohol), the procedures were much better tolerated by the patients, and there were no complications.

Adhesive capsulitis has been an elusive diagnosis for imaging specialists. Although it is often clinically obvious once limitation of motion develops ("frozen" shoulder) the patient can have pain for months prior to that time during which the diagnosis is not obvious and imaging may be called upon. In this study, Sridharan et al. [6] show that there are distinctive findings on PET/CT that allow one to make the diagnosis with relative confidence ( $87 \%$ specificity) Focal FDG uptake is seen either in the rotator interval, or the inferior joint capsule - the same location at which edema may be observed on MRI. Unfortunately, the authors do not tell us which stage of adhesive capsulitis the patients were in at the time of the scan. This observation is useful for several reasons: it fits with the general concept of the pathogenesis of the condition, it may be useful for diagnosis, and it may help to exclude more ominous conditions (neoplasm) for which PET CT is usually performed.

There has been a tremendous increase in surgical procedures to treat femoroacetabular impingement (almost fivefold, between 2005 and 2013). Whatever one may think about the validity of the postulated pathogenesis, it is likely that many radiologists will encounter patients who have undergone arthroscopic surgery to treat this condition. In this review [7], Dr. Crim describes and illustrates both the normal (sharply angulated concave contour at the lateral femoral head/neck junction - the "rim trim") and abnormal (incomplete or excessive resection of the cam lesion, instability, recurrent labral abnormalities following repair, cartilage damage and rapidly progressive osteoarthritis, psoas atrophy). Knowledge of the possible abnormalities must be very useful for the radiologist to ensure complete evaluation and accurate diagnosis, since as of the time of this writing, this was by far the most commonly downloaded article of the year.

An intriguing paper sheds light on the relationship between muscle strength (as suggested by cross-sectional area) and the risk of ACL tears. Wieschhoff and colleagues [8] measured the cross-sectional areas of both the vastus medialis and the semimembranosus muscles on the highest available axial image of a conventional knee MRI. They demonstrated that in patients who sustained a non-contact tear of the ACL, the quadriceps (vastus medialis) was disproportionately large compared to the hamstring (semimembranosus). This was not true of patients who sustained an injury that was due to direct contact. The authors observe that this is consistent with clinical studies that show that fatigue of the hamstring increases the load on the ACL and unopposed contraction of the quadriceps also results in strain upon the ACL.

\section{Evolving topics}

Although we think of most imaging studies as "noninvasive", injection of foreign substances can have unanticipated consequences. Recently, accumulation of gadolinium in the brain has been documented as the result of its use as an MRI contrast agent.

Gadolinium can also find its way into joints, either by direct injection, or following intravenous administration. Sharon Midura et al. [9] used chondrocyte cultures to investigate whether toxic effects were demonstrable at the doses used for arthrography. This is the same technique that was used to demonstrate the cartilage toxicity of long-acting local anesthetics. Fortunately, for the world of musculoskeletal imaging, no toxic effects were shown for doses up to $3 \mathrm{X}$ the typical arthrographic administration.

There has been considerable progress in both diagnosis and treatment of multiple myeloma, and consequently there is increasing need for imaging tools to measure progression of disease and the response to therapy. Therefore, a review article on this topic by Dutoit and Verstraete [10] was particularly welcome. While recognizing the value of $\mathrm{CT}$, including PETCT and whole-body CT, the authors focus their attention on the role of MRI, which they argue is intrinsically the most sensitive method available. They note that the finding of at 
least once unequivocal bone marrow lesion on MRI in a patient with smoldering myeloma should be a trigger for treatment. The authors turn their attention to the use of dynamic contrast-enhanced imaging, and pharmacokinetic modeling of contrast distribution. They note that the progression from MGUS to smoldering myeloma is accompanied by detectable increases in vascularity. Clinical response to therapy results in declines. Diffusion-weighted imaging can also be used to assess marrow cellularity, and response to treatment, although this approach is not yet a recommended part of the International Myeloma Working group consensus statement.

Finally, a review of ultrasound elastography [11] sheds light on this rapidly growing technique. The authors begin by reviewing the various technologies that have been developed in recent years, especially quasi-static (compression) elastography and shear wave elastography. Most attention is paid to shear wave elastography because it is the best method available that quantitatively reflects tendon stiffness. The authors discussion of the basic imaging methods helps to explain many of the recent clinical findings. They then proceed to describe the fundamental observations that have been made in the major tendons-Achilles, patella, and rotator cuff, as well as a few others.

\section{References}

1. Kang DJ, Lee SJ, Choo HJ, Her M, Yoon HK. Pancreatitis, panniculitis, and polyarthritis (PPP) syndrome: MRI features of intraosseous fat necrosis involving the feet and knees. Skelet Radiol. 2017;46(2):279-85.

2. Cowan S, Lozano-Calderon SA, Uppot RN, Sajed D, Huang AJ. Successful CT-guided cryoablation of phosphaturic mesenchymal tumor in the soft tissues causing tumor-induced osteomalacia. Skelet Radiol. 2017;46(2):273-7.

3. Pierce D, Kan JH, May M, Bisset GS. Pre-authorization processes have no effect on patients undergoing knee MRI in a pediatric setting when evaluated by specialists. Skelet Radiol. 2017;46(2): $171-5$.

4. Chew FS, Mulcahy MJ, Porrino JA, Mulcahy H, Relyea-Chew A. Prevalence of burnout among musculoskeletal radiologists. Skelet Radiol. 2017;46(4):497-506.

5. Chang CY, Kattapuram SV, Huang AJ, Simeone FJ, Torriani M, Bredella MA. Treatment of aneurysmal bone cysts by percutaneous CT-guided injection of calcitonin and steroid. Skelet Radiol. 2017;46(1):35-40.

6. Sridharan R. Focal uptake at the rotator interval or inferior capsule of shoulder on 18F-FDG PET/CT is associated with adhesive capsulitis. Skelet Radiol. 2017;46(4):533-8.

7. Crim J. Imaging evaluation of the hip after arthroscopic surgery for femoroacetabular impingement. Skelet Radiol. 2017;46(10): $1315-26$

8. Wieschhoff GG, Mandell JC, Czuczman GJ, Nikac V, Shah N, Smith SE. Acute non-contact anterior cruciate ligament tears are associated with relatively increased vastus medialis to semimembranosus cross-sectional area ratio: a case-control retrospective MR study. Skelet Radiol. 2017;46(11):1469-75.

9. Midura S, Schneider E, Rosen GM, Winalski CS, Midura RJ. In vitro chondrocyte toxicity following long-term, high-dose exposure to Gd-DTPA and a novel cartilage-targeted MR contrast agent. Skelet Radiol. 2017;46(1):23-33.

10. Dutoit JC, Verstraete KL. Whole-body MRI, dynamic contrastenhanced MRI, and diffusion-weighted imaging for the staging of multiple myeloma. Skelet Radiol. 2017;46(6):733-50.

11. Domenichini R, Pialat JB, Podda A, Aubry S. Ultrasound elastography in tendon pathology: state of the art. Skelet Radiol. 2017;46(12):1643-55. 